\title{
A J2EE based Management Information System for Higher Vocational Colleges
}

\author{
Bin $\mathrm{Lu}^{1}$, Changyu $\mathrm{Liu}^{2,3, *}$ and $\mathrm{Cong} \mathrm{Li}^{4}$ \\ ${ }^{1}$ School of Computer Science, Wuyi University, Jiangmen 529020, China \\ ${ }^{2}$ School of Computer Science and Engineering, South China University of Technology, \\ Guangzhou 510006, China \\ ${ }^{3}$ Department of Electrical and Computer Engineering, University of Miami, Coral Gables, \\ FL 33146, USA \\ ${ }^{4}$ College of Computer Science, Sichuan Normal University, Chengdu 610068, China \\ lbscut@gmail.com,yezhich@gmail.com (correspondingauthor),jkxy_lc@sicnu.edu.cn
}

\begin{abstract}
Informatization has always been one core competency for effective daily management. Although there are various types of management information systems available, few of them could take into account ever changing management requirements of higher vocational colleges. In this paper, we develop a J2EE based management information system for higher vocational colleges. Firstly, we perform an overview of the entire background and situation of the management informatization for higher vocational colleges. Secondly, according to requirement analysis and use case analysis, we divide the system, which requires three roles, i.e., college administrator, enterprise and graduate, into four functional modules, i.e., user login module, college administrator module, graduate module and enterprise module. Thirdly, for architecture design, we adopt a J2EE four-tier architecture, which are user presentation tier, control treatment tier, business logic tier and data persistence tier. Then, we introduce five entities, which are user information table, enterprise information table, graduate information table, recruitment information table and dynamic news table, for the database design. Furthermore, we also illustrate how we implement the final system.
\end{abstract}

Keywords: J2EE, management information system, higher vocational colleges

\section{Introduction}

Being already an indispensable part of Chinese modernization, the higher education plays an important role in its economic development and witnesses a fast popularization during recent years, resulting in a much faster growth of graduates than that of total social requirement. Taking up almost half of total annual graduates, the higher vocational graduates would probably choose to pursue advanced studies when they are exposed to an increasingly competitive employment environment. However, this is prejudicial to healthy development of labor market. Hence, the issue that how to adjust themselves to both complex employment environment and fierce employment market needs to be solved urgently.

Under this situation, most of higher vocational colleges would like to increase the employment rate by formulating employment policies and developing management information systems. However, their informatization progresses can't keep up with ever changing management requirements of higher vocational colleges. Besides, there is no such a versatile management information system that could be suitable for each higher vocational college.

In this paper, we design and implement a management information system for higher vocational colleges. Based on the J2EE four-tier architecture, the system is composed of 
three roles, four functional modules and five entities. Following the software engineering principle, we describe our system from four processes, which are system analysis, system design, system implementation and system application.

\section{System Analysis}

\subsection{Requirement Analysis}

Requirement analysis, as the first step the software lifecycle, could establish proper models by bridging the gap between project problems and their solutions. In software engineering, the requirement analysis involves those tasks that analyze the needs for a new product, consider possibly conflicting requirements from various stakeholders, document, validate and manage system requirements [1]. As one of the most important components in the requirement analysis, the functional requirement describes user requirements by many system functions that composed of inputs, behaviors and outputs. For the required management information system, major functions include a convenient management of higher vocational student information, an effective reduction of the management cost and an efficient modernized bridge among graduates, college administrators and enterprises.

Accordingly, we divide the required management information system into four functional modules, which are the user login module, the college administrator module, the graduate module, and the enterprise module. Specifically, for the user login module, the system should provide functions for selecting user roles, considering whether to adopt the IP login restrictions, and identifying the verification code. For the college administrator module, the system should provide functions for the acquisition of graduate information, the management of information distribution, the management of graduate archives, the statistic of information inquiry, and the system management. For the graduate module, the system should provide functions for the statistic of information inquiry, the management of personal information, and the featured alumni. For the enterprise module, the system should provide functions for the maintenance of enterprise information, the management of information distribution, the management of graduate archives, and the statistic of information inquiry.

\subsection{Use Case Analysis}

As a dynamic representation of system functions, the use case diagram involves actor, use case, and relationship between the actor and the use case. We can understand external functions of the system without implementation details from the use case diagram. The actor, which could play the role of persons, things, or systems, is an entity outside the system and participates in the process of several user cases. The use case is a set of scenes for describing how the actor achieves their goals by the system. There are mainly three roles in the management information system, which are the college administrator, the enterprise, and the graduate. Figure 1 shows intuitively the use case diagram of three roles based on functional requirements of the system.

\section{System Design}

Once we are familiar with system requirements and system use cases, we can then proceed to conduct detailed system design, which are the architecture design and the database design in this paper. 


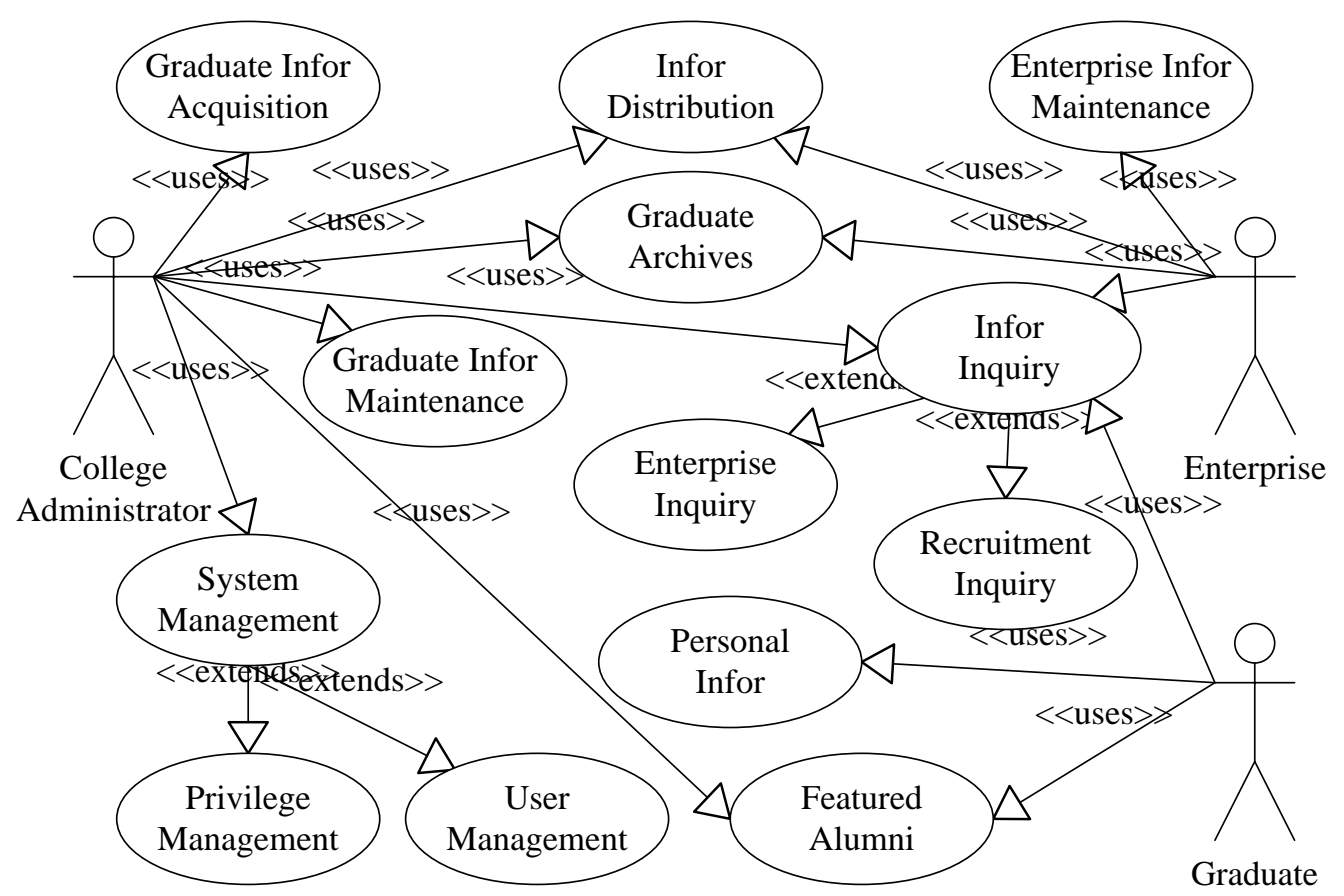

Figure 1. Use Case Diagram for the College Administrator, the Enterprise and the Graduate

\subsection{Architecture Design}

For architecture design, the J2EE provides a well known four-tier architecture, i.e. the client tier, the web tier, the enterprise JavaBean tier and the enterprise information system tier, which could integrates easily applications with other systems in a loosely coupled manner. In this paper, we adopt an improved J2EE four-tier architecture, as shown in Figure 2, which are the user presentation tier, the control treatment tier, the business logic tier and the data persistence tier, where upper tiers rely on lower tiers. Furthermore, we combine several technologies, such as JSP, Servlet, Struts and Apache, to implement the improved architecture.

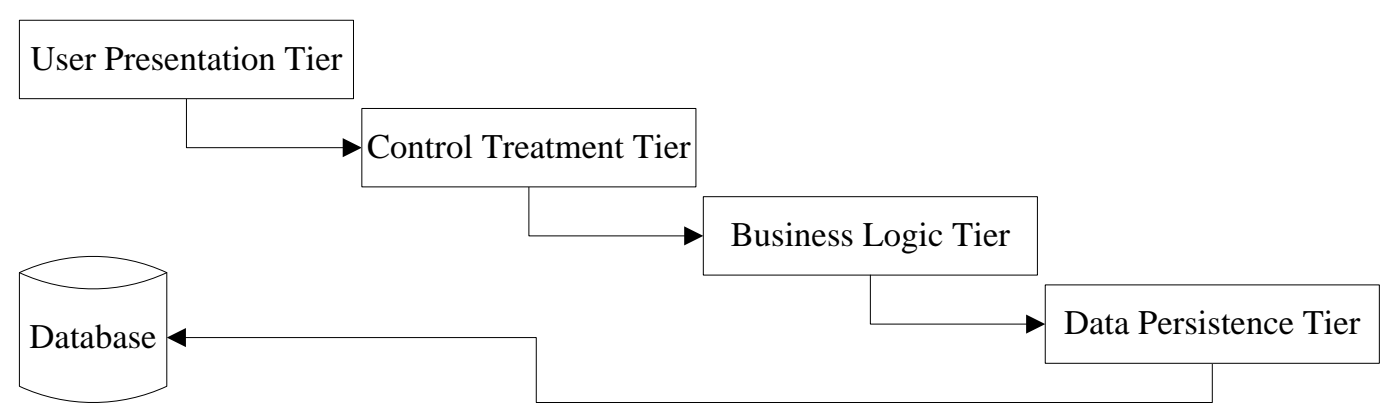

Figure 2. Four-tier Improved J2EE Architecture

\subsection{Database Design}

We can observe from the Figure 2 that the database is the fundament of the system. In this paper, we adopt the entity-relationship (ER) model based relational database. The ER model provides methods of entity types, properties and relationships to describe the real world based on the conceptual model, which is the initial stage of the database design. 
The ER relational database shows many advantages, such as high structured data, minimum redundancy, independence of procedures from data and easy expandability.

Accordingly, by using the Powerdesigner which is one kind of database design tools, we design an ER diagram, as shown in Figure 3, of the management information system based on the previous requirement analysis. We can perceive from this figure that there are five entities, which are the user information table USER_MIS4HVC, the enterprise information table ENTERPRISE_MIS4HVC, the graduate information table GRADUATE_MIS4HVC, the recruitment information table RECRUITMENT_MIS4HVC, and the dynamic news table DYNAMIC_NEWS_MIS4HVC.

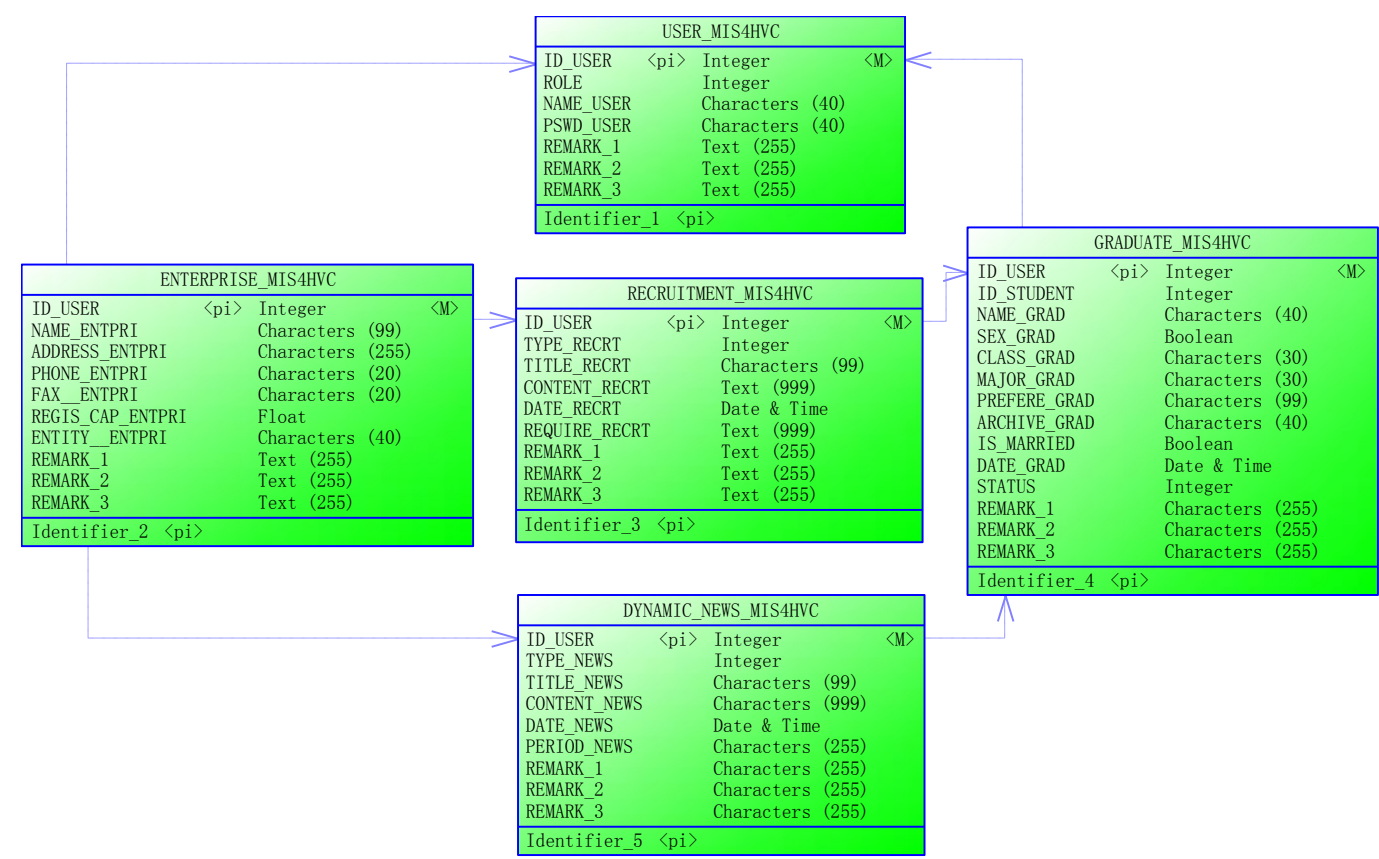

Figure 3. Entity-relationship (ER) Model

Once we fix the structure and the relationship of required entities by the ER model, we can then create these tables in SQL Server 2000 which is a relational database by using SQL script, as shown in Table 1.

\section{System Implementation}

We implement the management information system based on the improved J2EE fourtier architecture, which are the user presentation tier, the control treatment tier, the business logic tier and the data persistence tier. In this section, we illustrate how we implement each tier of the system.

Table 1. SQL Script for Creating ER Tables

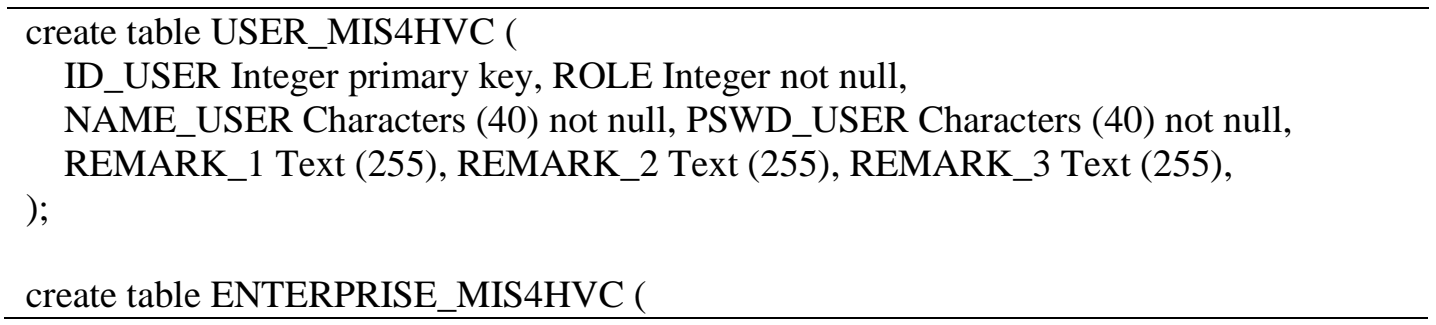




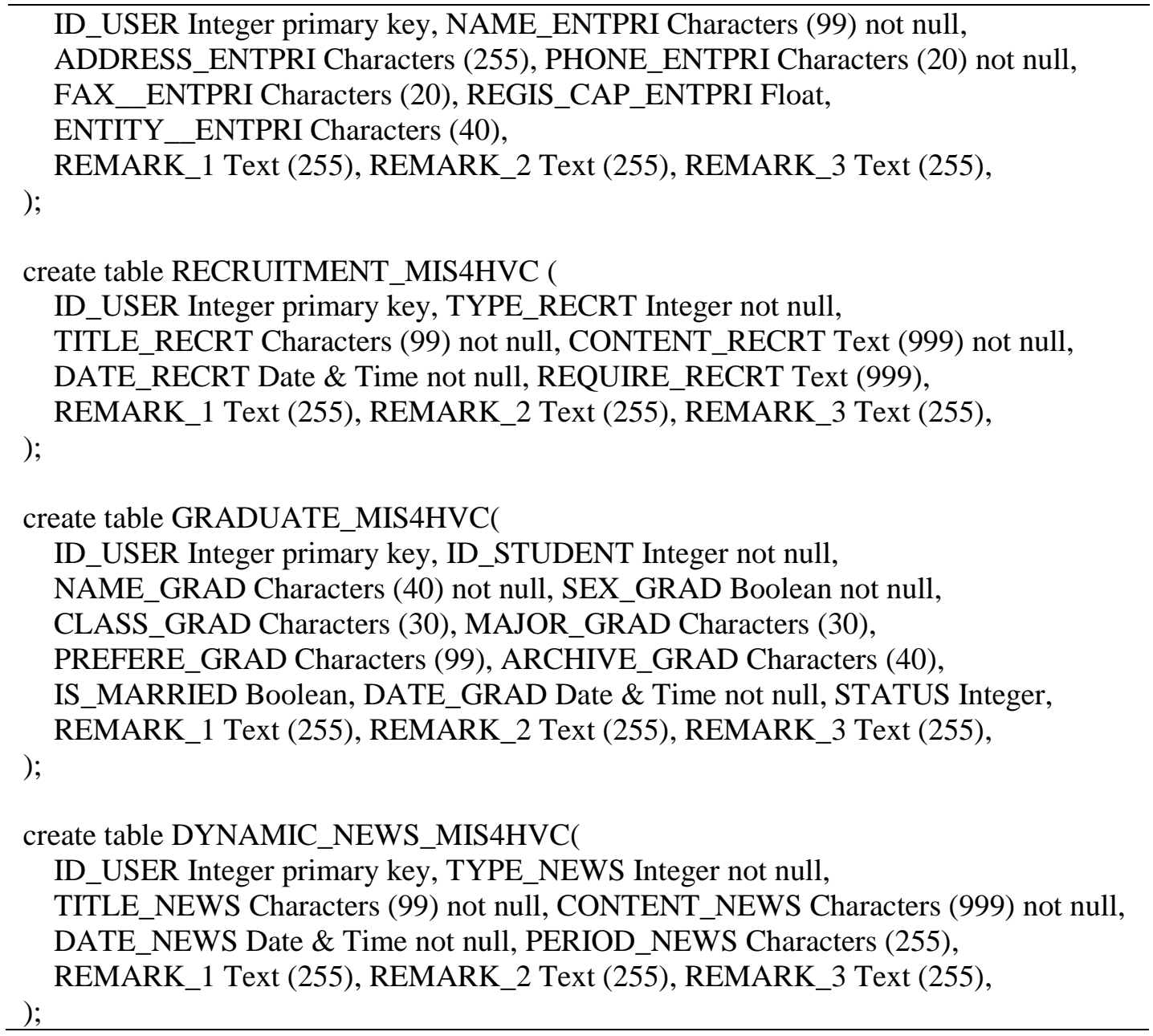

\subsection{The User Presentation Tier}

In order to separate the user presentation tier from the business logic tier, we substitute JSP page, JSP standard tag library (JSTL) and Strut 2 tag library for script codes of the page. Besides, we also show some implementation details about the Strut 2 tag library, the JSTL tag library and the JSP page.

Firstly, the Strut 2 tag library could provide abundant tags for the user presentation tier and the visual HTML editor for the JSP page, which could reduce heavily the amount of coding work for the application developer. From functional aspect, the Strut 2 tag library can be divided into two major categories, which are the non-UI tag library and the UI tag library, as shown in Figure 4. The non-UI tag library involves mainly the process control tag and the data access tag, whereas the UI tag library contains the form tag, the non-form tag and the Ajax tag. 


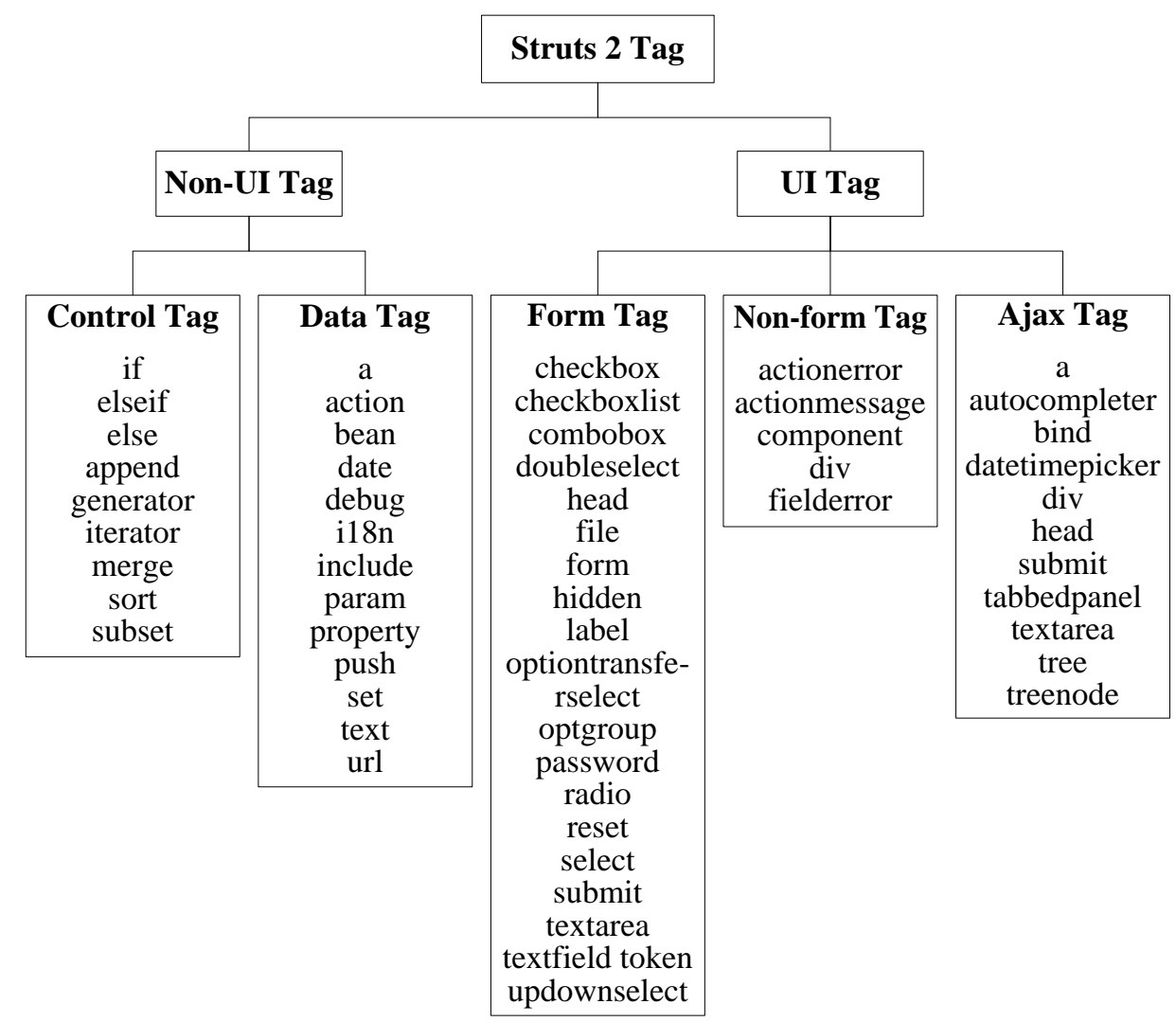

Figure 4. The Strut 2 Tag Library

Secondly, the JSTL is a customized tag library for common functions of the web applications, which include conditional judgment, data format management, XML operation and database access. Consisting of one tag library and one expression language (EL), the JSTL does not need to create the web page for reading XML and accessing database. At present, there are five subcategories of the JSTL, which are Core tag library, i18n tag library, SQL tag library, XML tag library, and functional tag library, as shown in Table 2.

Table 2. The JSTL Tag Library

\begin{tabular}{cccc}
\hline Sub Tag Libraries & URL & Prefixes & Examples \\
\hline Core tag library & http://java.sun.com/jstl/core & $\mathrm{c}$ & $<$ c:tagname...> \\
i18n tag library & http://java.sun.com/jstl/fmt & $\mathrm{fmt}$ & $<$ fmt:tagname...> \\
SQL tag library & http://java.sun.com/jstl/sql & $\mathrm{sql}$ & $<$ sql:tagname... \\
XML tag library & http://java.sun.com/jstl/xml & $\mathrm{x}$ & $<\mathrm{x}:$ tagname... \\
Functional tag library & http://java.sun.com/jstl/functions & $\mathrm{fn}$ & $<$ fn:tagname... \\
\hline
\end{tabular}

Thirdly, the JSP page, which could decouple views from the business logic tier, is used to display user interfaces, send client requests and receive server responses. There are mainly two types of JSP pages in the system, i.e. the system welcome page index.jsp and the user login page login.jsp. Firstly, defined in the Web.xml, as shown in Table 3, the index.jsp contains only one JSTL line <c:redirect url="u!login.z"/> for jumping to the login page login.jsp, where $u ! \operatorname{login} . z$ is equivalent to LoginAction.login(). The main purpose of the index.jsp is to use the role based access control model to verify the logged users based on the their user names and user passwords. There are mainly three roles in the management information system, which are the college administrator, the enterprise, 
and the graduate. Once one user is verified, the system would jump to /jsp/school/index.jsp by executing sc_u!login.z if the role is the college administrator, to /jsp/company/index.jsp by executing cmp_c!login.z if the role is the enterprise, and to /jsp/student/index.jsp by executing std_s!login.z if the role is the graduate.

\section{Table 3. The Mapping Relationship for Action u in struts.xml}

$$
\begin{aligned}
& \text { <global-results }>\quad<\text { result name="login" }>/ \text { jsp/login.jsp }\langle/ \text { result }>\langle/ \text { global-results }> \\
& <\text { action name }=" u \text { " class }=" l i n s . \text { school.action.LoginAction" }> \\
& <\text { result name }=" s c \_l g s u c c e s s " \text { type }=" \text { redirect" }>\text { sc_u!login.z }</ \text { result }> \\
& \left\langle\text { result name }=" \text { cmp_lgsuccess" type }=" \text { redirect" }>\mathrm{cmp} \_\mathrm{c} ! \operatorname{login} . \mathrm{z}\langle/ \mathrm{result}\rangle\right. \\
& \langle\text { result name="std_lgsuccess" type="redirect" }>\text { std_s!login.z }</ \text { result }> \\
& <\text { result name }=" \text { lgfail" type }=" \text { redirect" }>\text { u!login.z?tips }=\$\{\text { tips }\}</ \text { result }>
\end{aligned}
$$$$
</ \text { action }>
$$

\subsection{The Control Treatment Tier}

The Struts 2 controller is comprised of one key controller and several business controllers, i.e. Action. As a bridge between user requests and the business logics, the Action could locate Java classes of required business logics according to mapping relationships between received page data and Java classes that specified in the struts.xml file. For the key controller, we should configure both the web.xml file and the struts.xml file. For the business controllers, we should implement corresponding Action classes.

Firstly, as a general configuration file for Web applications, the web.xml can be used to set related filters, default home page and filter mapping relationship. Specifically, the setting of related filters is achieved by $<$ filter $><$ filter-name $>$ f_name $</$ filter-name $>$ $<$ filter-class $>$ f_class $</$ filter-class $></$ filter $>$, where f_name $\in\{$ struts-cleanup, sitemesh, struts 2$\}$ and f_class $\in\{$ ActionContextCleanUp, PageFilter, FilterDispatcher $\}$. The setting of default home page is implemented by <welcome-file-list $><$ welcomefile $>$ index.jsp $</$ welcome-file $></$ welcome-file-list $>$, where the index.jsp is the home page of the management information system. The setting of filter mapping relationship is realized by $<$ filter-mapping $><$ filter-name $>$ f_name $</$ filter-name $><$ url-pattern $>$ p_name $</$ url-pattern $></$ filter-mapping $>$, where $\mathrm{p}_{-}$name is the filter mode, such as $/ *$.

Secondly, as another general configuration file for Web applications, the struts.xml is the key component in the struts 2 architecture for forwarding all HTTP requests of the system. Both the global forwarding setting and the Action mapping relationship setting can be supported by the struts.xml file. For the global forwarding setting, we can use $<$ global-results $><$ result name $=$ l'ogin $» /$ jsp $/ \operatorname{login}$. jsp $</$ result $></$ global-results $>$ to jump to the $/$ jsp/login.jsp for any Actions with a return value of login. For the Action mapping relationship setting, Figure 5 shows four major Actions and their relationships of the management information system, which are $u, s c \_u, c m p \_c$ and std_s, where the identifier before the $>>$ denotes return value of the Action, the identifier after the $>>$ signify to jump to corresponding JSP page, $>>$ is the jumping operation. lins.school.action.LoginAction is the java class for the Login Action, lins.school.action.UserAction, lins.company.action.CompanyAction is the java class for the Company Action, lins.student.action.StudentAction is the java class for the Student Action, and S1-S5 are the implementation orders corresponding to different return values. 


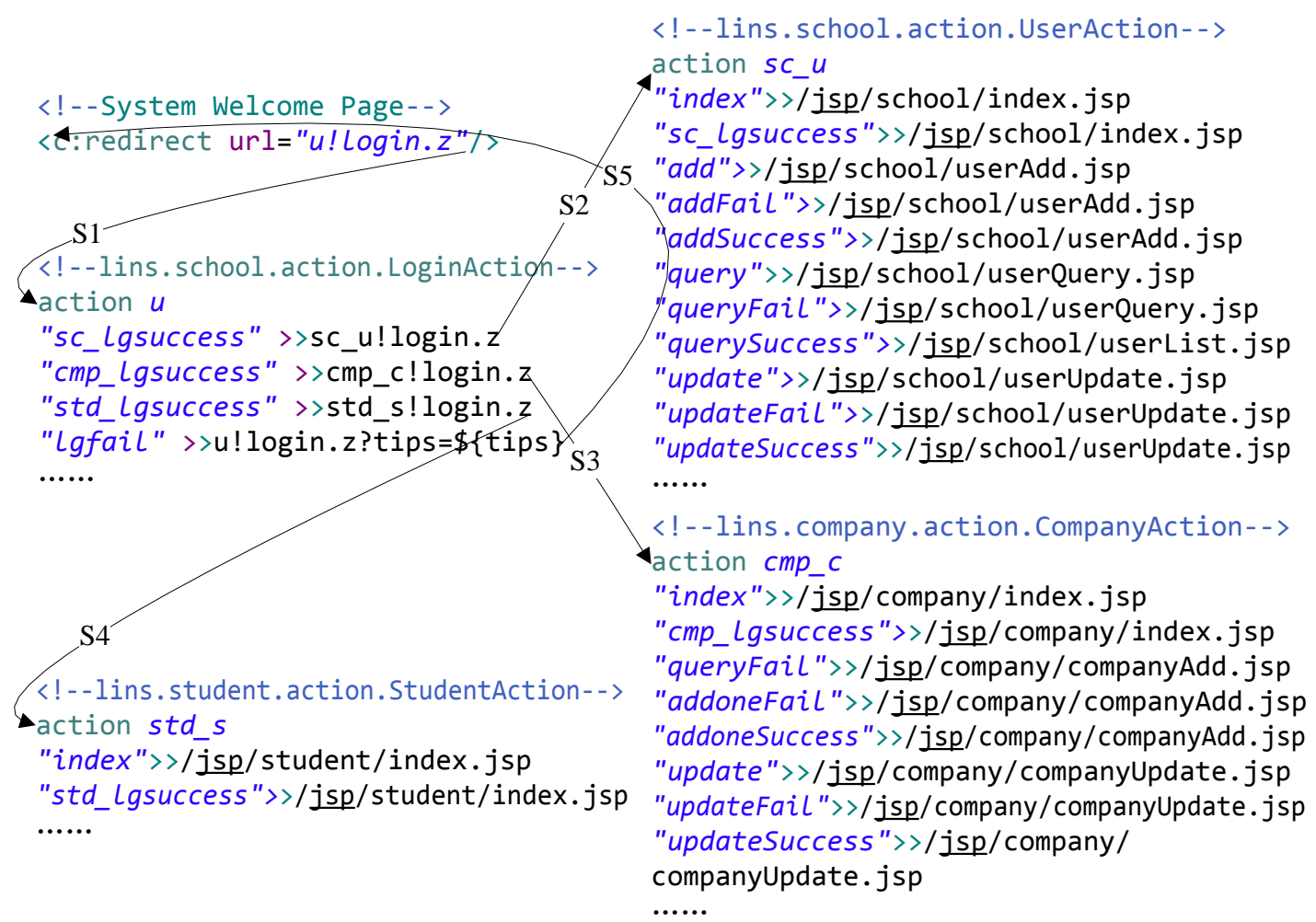

Figure 5. Four Major Actions and their Relationships

Thirdly, as business controller, the Action could call methods of its Java class and return the results to another Action or web page. To implement an Action, two types of definitions are required. Specifically, one of them is the definition of method execute() for responding user requests. The execute() is a method without any parameters and five return values, i.e. ERROR, INPUT, LOGIN, NONE and SUCCESS. The other is the definition of attributes and methods of set $\cdots()$ or get $\cdots()$ for receiving submitted parameters from users, where $\cdots$ should be substituted by the corresponding attribute name. For example, if the submitted parameters involve Username and Password, then the Action should contain methods of setUsername(), setPassword(), getUsername() and getPassword().

\subsection{The Business Logic Tier}

Providing functions for processing actual user requests, the business logic tier involves two types of classes, which are the business entity, i.e. the Entity of the ER model or the tables of the database, and the business Service. There are 11 such classes to be implemented in the business logic tier of the management information system, which are User, UserService, Company, CompanyService, Student, StudentService, JobInfo, JobInfoService, NewsInfo, NewsService, and ExcelService. Due to the limited space, we show only source java codes of the Student class in Table 4. Member variables of the Student class correspond to fields of the graduate information table GRADUATE_MIS4HVC. There are both a set $\cdots()$ method and a get $\cdots()$ method for setting and getting value of each field.

\subsection{The Data Persistence Tier}

The data persistence tier applies the Data Access Object (DAO), one standard J2EE design mode, to access the database. Data interfaces, i.e. methods to be used by system users, are defined in the DAO mode, which could separate low level data access 
operations from upper level business logic operations. Accessing mechanisms of various data sources, such as DBMS, LDAP and File, are provided in the DAO mode. One of the other advantages of the DAO mode is that developers are not need to understand details about how to operate the data source. In this paper, there are five java DAO classes, corresponding to five database tables, which are CompanyDao, JobDao, NewsDao, StudentDao and UserDao. Besides, final database operations, including database connection, create, read, update and delete (CRUD), of all DAO classes, are achieved by calling the fundamental java DB class.

Table 4. Source Java Codes for the Student Class

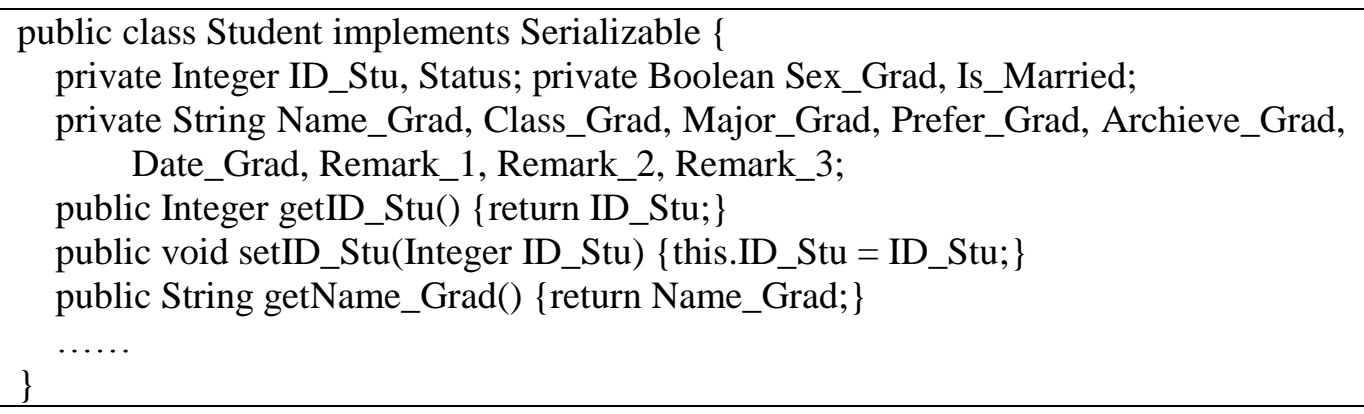

\section{System Application}

In this section, we use web pages of four functional modules in the management information system to display system applications.

\subsection{User Login Web Page}

There are three roles in the system, i.e., college administrator, graduate and enterprise. For all roles, the system provides a uniform login web page, where users could select their roles, input their user names, and input their passwords. Figure 6 shows the user login web page.

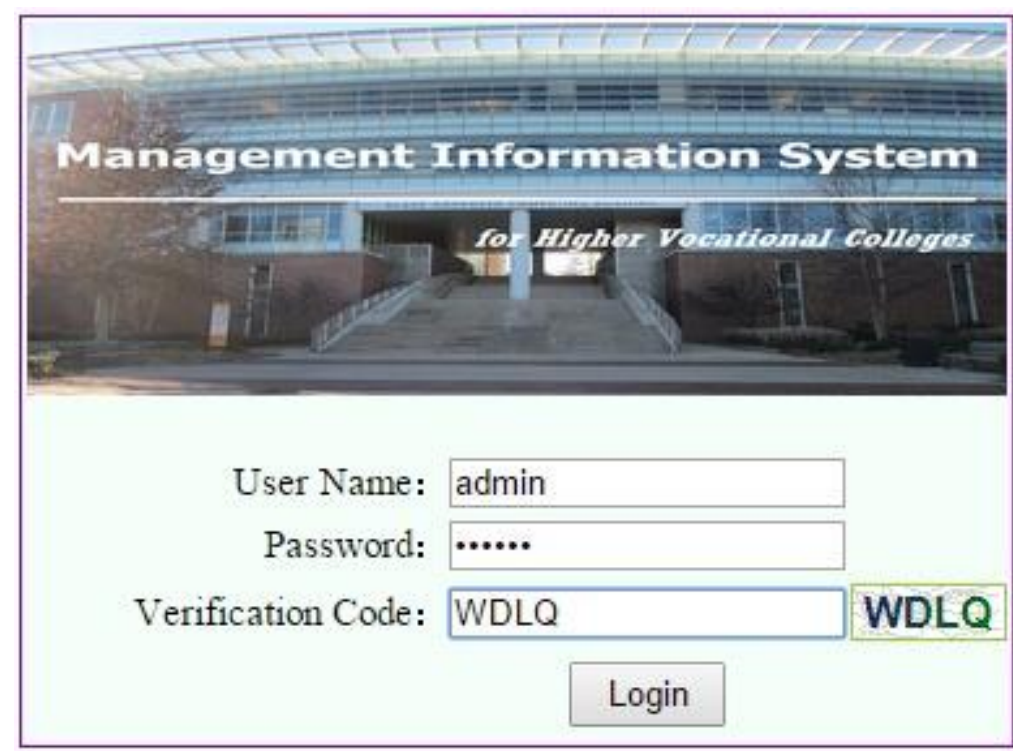

Figure 6.The User Login Web Page 


\subsection{College Administrator Web Page}

Once verified by the login web page, the college administrator could enter its own homepage. The acquisition of graduate information is one of primary functions provided by the web page. Furthermore, there are three acquisition ways, i.e., manual input, file import and information inquiry. Figure 7 shows the college administrator web page.

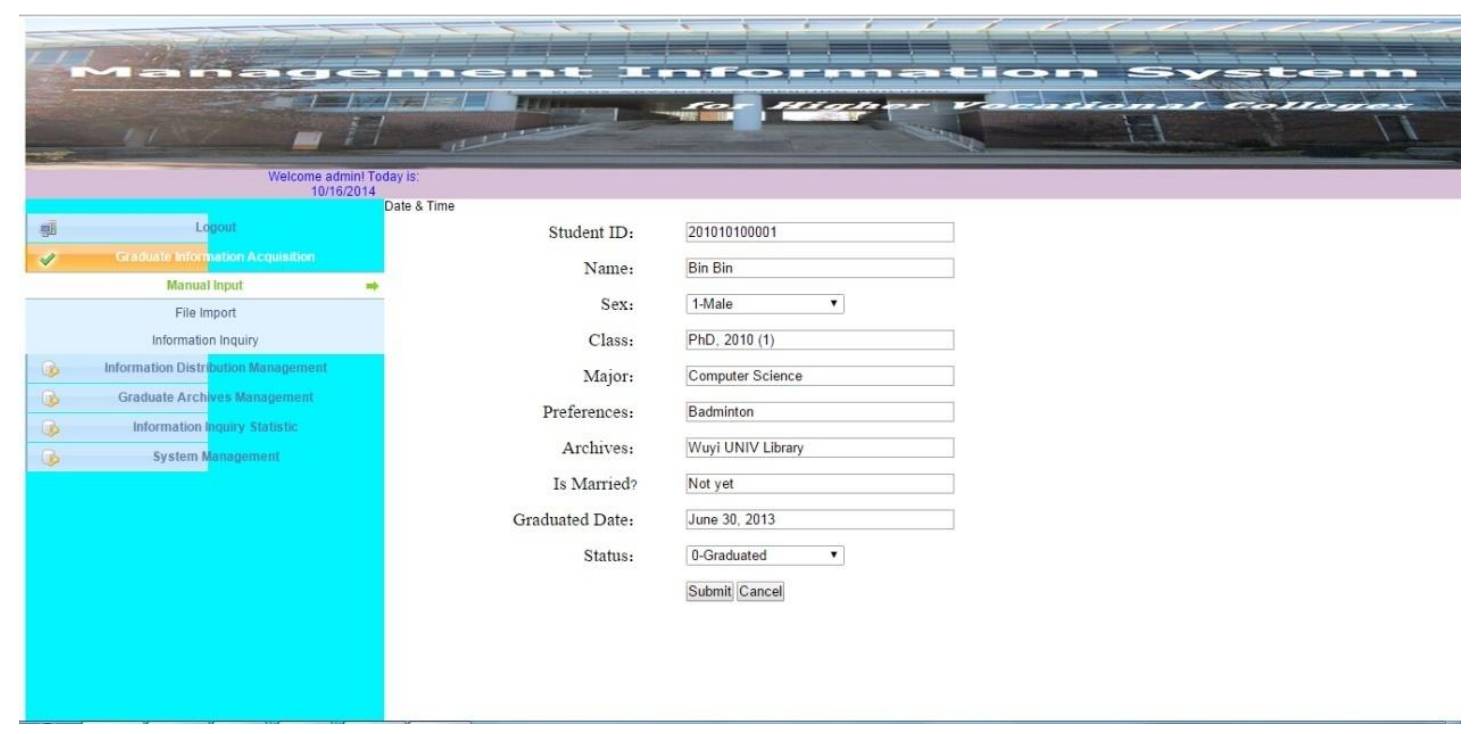

Figure 7.The College Administrator Web Page

\section{Conclusion}

Graduate of higher vocational colleges is one of the most important components in the entire employment structure. A reasonable management of graduate information is crucial to the economic development, the social stability and people essential benefits. In this paper, we describe how we develop a J2EE based management information system for higher vocational colleges from four aspects, which are system analysis, system design, system implementation and system application. At present, existing system applications have already demonstrated that the system could not only improve the employment rate and the management efficiency, but also bridge the gap among graduates, college administrators and enterprises, from technical aspects. In the future, we want to combine the SSH (Struts + Spring + Hibernate) framework with the current used J2EE four-tier architecture and strengthen system security by workflow or service oriented access control model.

\section{Acknowledgments}

This paper was supported by the Doctor Startup Foundation of Wuyi University under Grant No. 2014BS07, the Basic Theory and Scientific Research Project of Jiangmen City under title "Research on complex network evolution model and link prediction", and the National Natural Science Foundation of China under Grant No. 71202165. We would like to thank anonymous reviewers for helpful comments.

\section{References}

[1]. P. Herrmann and G. Herrmann, "Security requirement analysis of business processes", Electron, Commer, Res., vol. 6, no. 3-4, (2006), pp. 305-335.

[2]. Y. G. Jang, "Mobile colored overlays for people with visual stress", Int. J. Multimed. Ubiq., Eng., vol. 9, no. 6, (2014), pp. 25-30. 
[3]. J. X. Chen, "Modeling and performance analyzing of helix transmission base on Modelica", Key Eng. Mater., vol. 455, (2011), pp. 511-515.

[4]. M. Shuchao, F. Wei and G. Yang, "The policy thinking on the construction of modern vocational education system", Res. Educ. Dev., vol. 21, (2011), p. 4.

[5]. C. Liu, "Research on service-oriented and HLA-based simulation model of juice production line", In ICMTMA, vol. 3, (2010), pp. 167-170.

[6]. S. Dixit, L. Srivastava and G. Agnihotri, "Minimization of power loss and voltage deviation by SVC placement using GA", Int. J. Contr. Autom., vol. 7, no. 6, (2014), pp. 95-108.

[7]. H. J. Wang, "Structure design and multi-domain modeling for a picking banana manipulator", Adv. Mater. Res., vol. 97-101, (2010), pp. 3560-3564.

[8]. J. J. Powell and H. Solga, "Analyzing the nexus of higher education and vocational training in Europe: a comparative-institutional framework", Stud. Higher Educ., vol. 35, no. 6, (2010), pp. 705-721.

[9]. H. Li, "Catalytic hydrothermal pretreatment of corncob into xylose and furfural via solid acid catalyst", Bioresource Technol., vol. 158, (2014), pp. 313-320.

[10].X. Yang, L. Li and X. Fan, "A group mobility oriented history information based light location service", Int. J. Smart Home, vol. 8, no. 3, (2014), pp. 33-48.

[11].M. Kuijpers and F. Meijers, "Learning for now or later? career competencies among students in higher vocational education in the Netherlands", Stud. Higher Educ., vol. 37, no. 4, (2012), pp. 449-467.

[12].H. Wang, "Study on behavior simulation for picking manipulator in virtual environment based on binocular stereo vision", In ICSC, (2008), pp. 27-31.

[13].H. Bedoui, N. Bouguila and K. B. Othman, "Use of sensitivity method for the detection and the localization of linear system defect", Int. J. Contr. Autom., vol. 7, no. 5, (2014), pp. 17-34.

[14].B. Lu, "Discovery of community structure in complex networks based on resistance distance and center nodes", J. Comput. Inf. Syst., vol. 8, no. 23, (2012), pp. 9807-9814.

[15].H. O. N. G. Zhen-yin, "Some problems and reflections on school-enterprise in-depth cooperation in vocational education", J. Higher Educ., vol. 3, (2010), p. 10.

[16].Y. Gu, X. Wu and J. Wang, "A simplification method of terrain modeling based on spatialautocorrelation", Int. J. Multimed. Ubiq. Eng., vol. 9, no. 5, (2014), pp. 185-194.

[17].C. Y. Liu, "A TPSAC model and its application to mechanical cloud simulation”, Int. J. Secur. Appl., vol. 8, no. 1, (2014), pp. 45-56.

[18].S. Park, S. Oh and K. Lee, “Analysis of common security factor on energy industry”, Int. J. Smart Home, vol. 8 , no. 2, (2014), pp. 115-122.

[19].C. Barone and L. Ortiz, "Over education among European university graduates: a comparative analysis of its incidence and the importance of higher education differentiation", Higher Educ., vol. 61, no. 3, (2011), pp. 325-337.

[20].H. L. Li, "One-step heterogeneous catalytic process for the dehydration of xylan into furfural", BioResources, vol. 8, no. 3, (2013), pp. 3200-3211.

[21].N. Kang and H. Morita, "The acquisition of the thematic roles of the English verb open by college students", Int. J. Smart Home, vol. 8, no. 2, (2014), pp. 153-168.

[22].R. S. Bhadoria, G. S. Tomar and S. Kang, "Proficient energy consumption aware model in wireless sensor network", Int. J. Multimed. Ubiq. Eng., vol. 9, no. 5, (2014), pp. 27-36.

[23].C. Liu, "Modeling physical and chemical growths of avascular tumor", Int. J. Multimed. Ubiq. Eng., vol. 9, no. 4, (2014), pp. 349-362.

[24].S. Marginson, "Higher education in east Asia and Singapore: rise of the Confucian model", Higher Educ., vol. 61, no. 5, (2011), pp. 587-611.

[25].H. J. Wang, "Study on a location method for bio-objects in virtual environment based on neural network and fuzzy reasoning", In ICIRA, vol. 5928, (2009), pp. 1004-1012.

[26].Y. A. Li, J. Whalley, S. Zhang and X. Zhao, "The higher educational transformation of China and its global implications”, World Econ., vol. 34, no. 4, (2011), pp. 516-545.

\section{Authors}

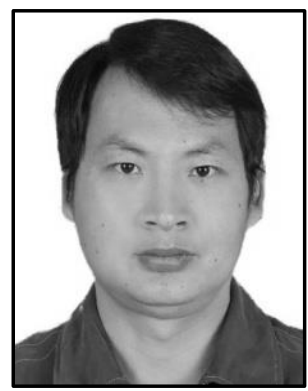

Bin Lu is currently a lecturer in the School of Computer Science at Wuyi University. He received his Ph.D. degree in 2013 from South China University of Technology. He is a reviewer of the Journal of Yangtze River Scientific Research Institute since 2010. His main research interests include complex network and machine learning. 


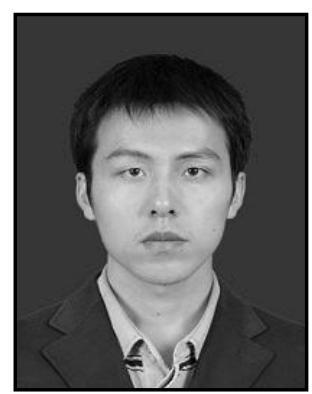

Changyu Liu joined the Communication and Computer Network Lab of Guangdong as a PhD student in 2010 at South China University of Technology, advised by Prof. Shoubin Dong. He was a Visiting Scholar at the School of Computer Science, Carnegie Mellon University, from September 2012 to October 2013, advised by Dr. Alex Hauptmann. Then, he worked with Prof. Mohamed Abdel-Mottaleb at the Department of Electrical and Computer Engineering, University of Miami, from October 2013 to September 2014. His research interests include computer vision and machine learning.

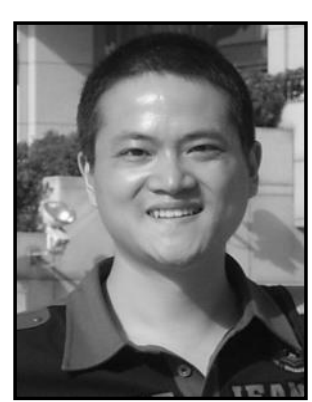

Cong $\mathbf{L i}$ received the $\mathrm{PhD}$ degree in Management Science and Engineering from Hefei University of Technology in 2009. He is an associate professor at the College of Computer Science, Sichuan Normal University. His research interests include E-commerce and business intelligence. He is a member of the ACM, and also is a senior member of the China Computer Federation. 